\title{
Left Atrial Enlargement on Transthoracic Echocardiography Predicts Left Atrial Thrombus on Transesophageal Echocardiography in Ischemic Stroke Patients
}

\author{
James Anaissie, ${ }^{1}$ Dominique Monlezun, ${ }^{1}$ A. Seelochan, ${ }^{2}$ James E. Siegler, ${ }^{1}$ \\ Maria Chavez-Keatts, ${ }^{3}$ Jonathan Tiu, ${ }^{1}$ Denise Pineda, ${ }^{1}$ Alexander George, ${ }^{1}$ Amir Shaban, ${ }^{1,4}$ \\ Nidal Abi Rafeh, ${ }^{5}$ Laurie Schluter, ${ }^{1}$ Sheryl Martin-Schild, ${ }^{1}$ and Ramy El Khoury ${ }^{1}$ \\ ${ }^{1}$ Tulane Stroke Research Program, Tulane University Medical Center, New Orleans, LA, USA \\ ${ }^{2}$ Meharry Medical College, Nashville, TN, USA \\ ${ }^{3}$ Department of Neurology, Louisiana State University Health Sciences Center, New Orleans, LA, USA \\ ${ }^{4}$ Department of Neurology, University of Iowa, Iowa City, IA, USA \\ ${ }^{5}$ Department of Cardiology, Tulane University Medical Center, New Orleans, LA, USA
}

Correspondence should be addressed to Ramy El Khoury; relkhoury@neurocareoflouisiana.com

Received 5 July 2016; Accepted 25 September 2016

Academic Editor: Ramazan Akdemir

Copyright (c) 2016 James Anaissie et al. This is an open access article distributed under the Creative Commons Attribution License, which permits unrestricted use, distribution, and reproduction in any medium, provided the original work is properly cited.

\begin{abstract}
Background. Transesophageal echocardiogram (TEE) is superior to transthoracic echocardiogram (TTE) in detecting left atrial thrombus (LAT), a risk factor for stroke, but is costly and invasive, carrying a higher risk for complications. Aims. To determine the utility of using left atrial enlargement (LAE) on TTE to predict LAT on TEE. Methods. AIS patients who presented in 06/2008-7/2013 and underwent both TTE and TEE were identified from our prospective stroke registry. Analysis consisted of multivariate logistic regression with propensity score adjustment and receiver operating characteristic (ROC) area under the curve (AUC) analyses. Results. 219 AIS patients underwent both TTE and TEE. LAE on TTE was detected in 113 (51.6\%) of AIS patients. Patients with LAE on TTE had higher proportion of LAT on TEE ( $8.4 \%$ versus $1.0 \%, p=0.018$ ). LAE on TTE predicted increased odds of LAT on TEE $(\mathrm{OR}=8.83,95 \%$ CI $1.04-74.83, p=0.046)$. The sensitivity and specificity for LAT on TEE by LAE on TEE were $88.89 \%$ and $52.20 \%$, respectively (AUC $=0.7054,95 \%$ CI 0.5906-0.8202). Conclusions. LAE on TTE can predict LAT detected on TEE in nearly $90 \%$ of patients. This demonstrates the utility of LAE on TTE as a potential screening tool for LAT, potentially limiting unneeded costs and complications associated with TEE.
\end{abstract}

\section{Introduction}

Approximately one in four acute ischemic strokes (AIS) can be attributed to an embolism that originates in the heart $[1,2]$. In particular, cardiac morphology and rhythm changes have been documented as chief contributing factors for thrombus formation [3-6]. The left atrium is the most common chamber in which these thrombi originate, and thrombus formation is strongly associated with the pathophysiology of atrial fibrillation $[4,5]$. The disease process leads to rhythmic and structural changes within the heart, with left atrium dilation and left atrium appendage (LAA) dysfunction being prominent contributing factors to thrombus formation in these patients [5].

As left atrial enlargement (LAE) is thought to be a structural precursor of atrial fibrillation, accurate detection of this cardiac abnormality can aid as a screening modality for prevention of recurrent AIS. To properly assess such cardiac changes, echocardiography serves an important diagnostic tool for at-risk patients. However, the decision to perform transesophageal echocardiography (TEE) after routine transthoracic echocardiography (TTE) in AIS is 
TABLE 1: Indications for transthoracic echocardiography versus transesophageal imaging for embolism detection (adapted from [12]).

\begin{tabular}{ll}
\hline TTE & TEE \\
\hline $\begin{array}{l}\text { Patients } \geq 45 \text { years with a neurologic event and no identified } \\
\text { cerebrovascular disease }\end{array}$ & $\begin{array}{l}\text { Patients }<45 \text { years without known cardiovascular disease (i.e., } \\
\text { absence of infarction or valvular disease history) } \\
\text { Any patient with an abrupt occlusion of a major peripheral or } \\
\text { visceral artery }\end{array}$ \\
$\begin{array}{l}\text { Patients with a high pretest probability of a cardiac embolic source } \\
\text { in whom a negative TTE would be likely to be falsely negative } \\
\text { Patients with atrial fibrillation and suspected left atrial or left atrial } \\
\text { appendage thrombus, especially in the absence of therapeutic } \\
\text { anticoagulation }\end{array}$ \\
$\begin{array}{l}\text { Patients in whom TEE is contraindicated (e.g., esophageal } \\
\text { stricture, unstable hemodynamic status) or who refuse TEE }\end{array}$ & $\begin{array}{l}\text { Patients with a mechanical heart valve } \\
\text { Patients with suspected aortic pathology }\end{array}$
\end{tabular}

controversial. Table 1 lists some of the common indications for choosing one of the two echocardiographic imaging modalities.

Prior literature and clinical practice have recognized the cost-effectiveness and risk TEE or TTE in patients with suspected cardiac disease $[3,4]$. TTE is noninvasive and less expensive than TEE; conversely, TEE is superior to TTE in detecting cardiac structural abnormalities and intracardiac thrombi $[3,4]$. Specifically, TEE is thought to be superior at quantifying the degree of LAE and visualizing the LAA. As both the LAE and LAA are considered to be important sites and/or precursors of thrombi formation in cardioembolic stroke, TEE is considered to have a higher utility for patients who are at high risk for AIS (such as prior history of embolic stroke, AF, and valvular abnormalities). Further complicating the decision making between these modalities is the fact that TEE is not tolerated by all patients and carries a higher risk of inpatient complications-including bleeding and transient cardiac arrhythmias-which may lead to extended hospital stay [6].

Due to the potential aforementioned disadvantages found in TEE, it is worthwhile to compare the diagnostic and/or screening value of TTE versus TEE. In this study, we sought to determine the utility of TTE imaging in AIS patients. Our first analysis centers around the use of TTE to diagnose LAE in these patients as opposed to the standard TEE imaging. Secondly, the ability for TTE to detect left atrial thrombus (LAT) within AIS patients that have evidence of LAE will be evaluated by comparing against patients who had a LAT found on TEE (the gold standard for detecting LAT).

\section{Methods}

2.1. Study Population. We conducted a single-center retrospective analysis of all consecutive patients who presented with AIS between July 2008 and July 2013 [7]. Patients who did not have both TTE and TEE were excluded. Stroke etiology was defined according to the Trial of Org 10172 in Acute Stroke Treatment [8]. Patient demographics, past medical history, and imaging characteristics were all collected prospectively in our stroke registry. All patients were assessed by staff stroke neurologists or stroke fellows at a single academic emergency department.
2.2. Definitions. Patients that were included within the retrospective analysis were classified as having LAE on the basis of left atrial volume to body surface area measurements noted from TTE and TEE imaging. As per recommendations from the American Society of Echocardiography, the ratio of left atrial volume to body surface area was used for the evaluation on TTE. For TEE, evaluation and classification were done on the basis of the biplane area formula noted in Lang et al. [9]. The patients were divided into 2 groups: (1) no LAE (LA volume to BSA ratio $<34 \mathrm{~mL} / \mathrm{m}^{2}$ on TTE) and (2) LAE (moderate LAE noted to have measured ratios of 34$39 \mathrm{~mL} / \mathrm{m}^{2}$ on TTE; severe LAE defined as LA volume to BSA ratio $>39 \mathrm{~mL} / \mathrm{m}^{2}$ as per Tsang et al.) [10].

2.3. Statistical Analyses. The patient demographics, past medical history, and imaging characteristics were compared between two groups (Table 2). Namely, AIS patients with evidence of LAE on the screening TTE $(n=113)$ were compared against their counterparts without LAE identified via TTE $(n=106)$. To maintain standardization between the two groups, the individual's chief clinician was chiefly responsible for guiding the decision to obtain both diagnostic tests. Subsequently, both of the analyzed groups were narrowed specifically to patients who underwent both TTE and TEE. The comparative metrics were in line with criteria used for choosing either TTE or TEE as an echocardiographic modality [11, 12].

Categorical data, presented as proportions, were analyzed using Pearson Chi-Square or Fisher exact test where appropriate. Continuous data, presented as medians and ranges, were compared using Wilcoxon Rank Sum tests. Univariate logistic regression was used to assess the odds of predicting LAT on TEE for each group. Receiver operator characteristic (ROC) curves were generated to assess the ability of LAE on TTE to predict LAT on TEE. No adjustments were made for multiple comparisons as this was an exploratory analysis.

Propensity score (PS) matching was conducted based on the likelihood of receiving TEE among TTE subjects to control for the confounding effect of baseline patient traits associated with receiving TEE and TTE. The PS was created based on documented predictors of receiving TEE, regardless of their statistical significance on bivariate analysis, to create 
TABLE 2: Clinical and demographic characteristics of AIS patients with and without left atrial enlargement on transthoracic echocardiogram.

\begin{tabular}{|c|c|c|c|}
\hline & $\begin{array}{l}\text { AIS subjects without LAE on TTE } \\
n=106(48.4 \%)\end{array}$ & $\begin{array}{l}\text { AIS subjects with LAE on TTE } \\
\qquad n=113(51.6 \%)\end{array}$ & $p$ value \\
\hline Age, median (range) & $59(26-83)$ & $64(26-90)$ & $<0.001$ \\
\hline African American, number (\%) & $74(71.15 \%)$ & $73(66.36 \%)$ & 0.450 \\
\hline \multicolumn{4}{|l|}{ Past Medical History, number (\%) } \\
\hline Atrial fibrillation & $4(3.81 \%)$ & $11(10.00 \%)$ & 0.075 \\
\hline Chronic heart failure & $2(1.94 \%)$ & $19(18.81 \%)$ & $<0.001$ \\
\hline Diabetes & $25(24.51 \%)$ & $40(37.04 \%)$ & 0.050 \\
\hline Hypertension & $72(68.57 \%)$ & $95(86.36 \%)$ & 0.002 \\
\hline Coronary artery disease & $16(15.09 \%)$ & $27(24.77 \%)$ & 0.076 \\
\hline Known prior stroke & $35(33.33 \%)$ & $39(35.78 \%)$ & 0.707 \\
\hline Hyperlipidemia & $31(29.52 \%)$ & $42(38.18 \%)$ & 0.180 \\
\hline Carotid stenosis & $0(0.00 \%)$ & $0(0.00 \%)$ & NA \\
\hline Active smoker & $43(41.75 \%)$ & $40(36.70 \%)$ & 0.451 \\
\hline
\end{tabular}

Definitions: LAE, left atrial enlargement; TTE, transthoracic echocardiogram.

a parsimonious probit regression model predicting treatment $[13,14]$.

Multivariate logistic regression with adjustment for the PS was then performed for LAE on TTE predicting LAT on TEE. Adjustment is a popular PS technique in stroke outcome research that has been shown to be similar to other PS techniques of matching and weighting [15]. Outcome results were then compared to average treatment effect on the treated (ATT) between PS-matched subjects using Kernel matching with imposition of common support $[16,17]$.

\section{Results}

Among the 219 AIS patients who had both TTE and TEE performed, LAE on TTE was detected in 113 patients $(51.6 \%)$. Patients with LAE on TTE were more likely to be older (64 years versus 59 years, $p<0.001)$ and were more likely to have a history of chronic heart failure (18.81\% versus $1.94 \%, p<$ $0.001)$ or hypertension $(86.36 \%$ versus $68.57 \%, p=0.002)$ when compared to patients with normal left atrial size.

Table 3 displays the differences in TEE findings for the two study groups. Patients with LAE on TTE exhibited higher median left atrial volume to BSA ratio compared to patients without LAE on TTE $\left(4.2 \mathrm{~mL} / \mathrm{m}^{2}\right.$ versus $3.4 \mathrm{~mL} / \mathrm{m}^{2}, p<$ 0.001). Patients with LAE on TTE were more likely to have evidence of valvular disorders, which included mitral valve regurgitation ( $54.86 \%$ versus $30.18 \%, p<0.001$ ), mitral annular calcification (46.81\% versus $21.43 \%, p<0.001$ ), or aortic valve stenosis (31.25\% versus $16.04 \%, p=0.004)$. Patients with LAE on TTE were also more likely to have left ventricular dilatation $(17.02 \%$ versus $4.04 \%, p=0.003)$ and left ventricular hypertrophy (54.93\% versus $19.12 \%, p<$ 0.001 ) on TEE than patients without LAE.

Among the entire sample, TTE failed to detect LAT whereas 9 patients $(4.11 \%)$ had LAT on TEE. Among patients with both TTE and TEE documenting presence or absence of LAE, LAE was detected in 89 patients by TTE and 76 by TEE, with LAE detected by both TTE and TEE in 63

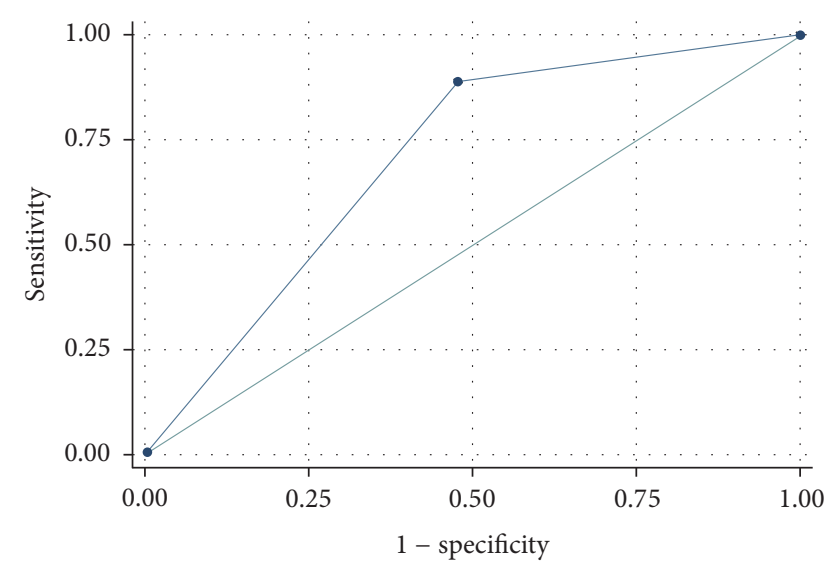

FIGURE 1: Receiver operating characteristic curve of left atrial enlargement on transthoracic echocardiogram classifying left atrial thrombus on transesophageal echocardiogram. Area under curve $=$ 0.7054 (95\% CI 0.5906-0.8202).

(70.79\%) patients, TTE alone in 26 (29.21\%), and TEE alone in $13(15.29 \%)$, as seen in Figure 2. As a result, TTE was 89.68\% sensitive for detecting LAE whereas TEE was $57.94 \%$ sensitive. Moreover, the patients who showed evidence of LAE on TTE were more likely to show signs of thrombus on TEE imaging (8.4\% versus $1.0 \%, p=0.018)$.

ATT results of PS-matched subjects showed a similarly increased likelihood of LAT when LAE was detected using TTE $(7.5 \%, T$-stat $=2.12, p<0.05)$. Within the subgroup of patients who displayed evidence of LAE on TTE $(n=113)$, $8(8.42 \%)$ had LAT on TEE. Therefore, TEE conferred a PPV value of $92.9 \%(8 / 9)$ in detecting LAT. Conversely, the absence of a LAE on TTE correlated with NPV of $99 \%$ for detecting LAT (105/106).

The ROC curve for LAE on TTE predicting LAT is displayed in Figure 1. The finding of LAE on TTE predicted LAT with a sensitivity of $88.9 \%$ and specificity of $52.2 \%$ $(\mathrm{ROC}=0.7054,95 \%$ CI 0.5906-0.8202). Additionally, 
TABLE 3: Transesophageal echocardiographic determination of cardiac characteristics of AIS patients (results stated with number of patients observed with noted characteristic on TEE; percentage noted subsequently is a comparison to the number of patients who have a certain characteristic on TTE).

\begin{tabular}{|c|c|c|c|}
\hline & $\begin{array}{l}\text { AIS subjects without LAE on TTE } \\
\qquad n=106(48.4 \%)\end{array}$ & $\begin{array}{l}\text { AIS subjects with LAE on TTE } \\
\qquad n=113(51.6 \%)\end{array}$ & $p$ value \\
\hline Left atrial size, median (range), $\mathrm{mL} / \mathrm{m}^{2}$ & $3.4(2.3-13.0)$ & $4.2(2.7-6.7)$ & $<0.001$ \\
\hline Left ventricular size, median (range), $\mathrm{mL} / \mathrm{m}^{2}$ & $89(44-303)$ & $98(31.6-1333)$ & 0.081 \\
\hline TTE detection of LAT, \% & $0(0.00 \%)$ & $0(0.00 \%)$ & NA \\
\hline $\mathrm{PFO}, \%$ & $1(1.32 \%)$ & $3(3.85 \%)$ & 0.324 \\
\hline ASD, \% & $29(27.89 \%)$ & $30(28.30 \%)$ & 0.052 \\
\hline Interatrial shunt, $\%$ & $5(5.95 \%)$ & $10(11.24 \%)$ & 0.217 \\
\hline Mitral valve regurgitation, $\%$ & $32(30.18 \%)$ & $62(54.86 \%)$ & $<0.001$ \\
\hline Mitral valve prolapse, $\%$ & $0(0.00 \%)$ & $2(3.12 \%)$ & 0.136 \\
\hline Mitral annular calcification, $\%$ & $18(21.43 \%)$ & $44(46.81 \%)$ & $<0.001$ \\
\hline Left ventricular dilatation, $\%$ & $4(4.04 \%)$ & $16(17.02 \%)$ & 0.003 \\
\hline Left ventricular hypertrophy, \% & $13(19.12 \%)$ & $19(54.93 \%)$ & $<0.001$ \\
\hline Diastolic dysfunction, $\%$ & $59(62.11 \%)$ & $71(83.53 \%)$ & 0.001 \\
\hline Aortic valve regurgitation, $\%$ & $28(26.42 \%)$ & $42(38.19 \%)$ & 0.230 \\
\hline Aortic valve stenosis, $\%$ & $17(16.04 \%)$ & $35(31.25 \%)$ & 0.004 \\
\hline TEE LAE, \% & $13(15.29 \%)$ & $63(70.79 \%)$ & $<0.001$ \\
\hline TEE detection of LAT, \% & $1(1.04 \%)$ & $8(8.42 \%)$ & 0.016 \\
\hline
\end{tabular}

Key: LAE, left atrial enlargement; PFO, patent foramen ovale; ASD, atrial septal defect; TTE, transthoracic echocardiogram; TEE, transesophageal echocardiogram; LAT, left atrial thrombus; NA, not applicable.

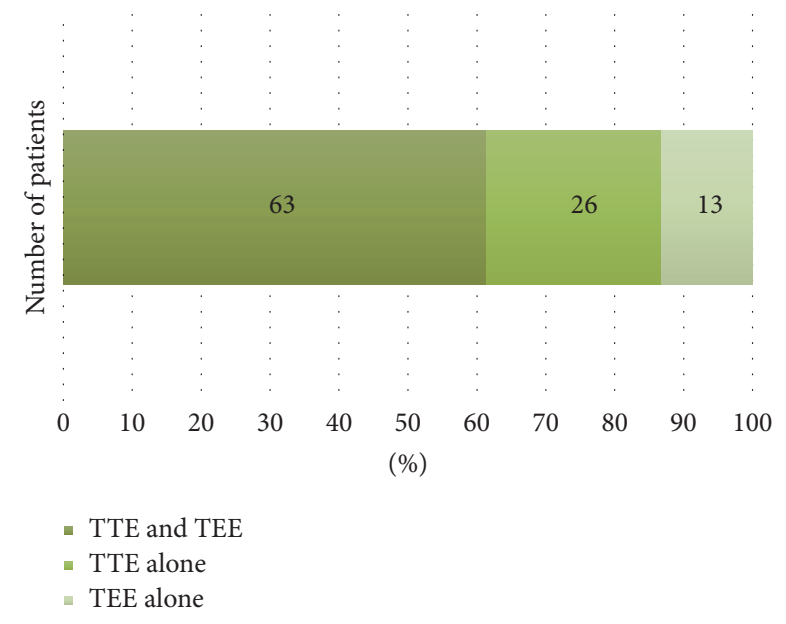

FIGURE 2: Number of patients demonstrating left atrial enlargement on each echocardiographic imaging modality. TTE $=$ transthoracic echocardiogram; TEE = transesophageal echocardiogram.

multivariate logistic regression with adjustment for the PS revealed that LAE on TTE was associated with an increased odds of LAT detection $(\mathrm{OR}=8.83,95 \%$ CI 1.04-74.83, $p=$ $0.046)$.

\section{Discussion}

The results of our study indicate that a normal left atrium on TTE practically excludes the possibility of thrombus formation in this cardiac chamber. As the finding of LAE on
TTE was almost $90 \%$ sensitive for predicting LAT on TEE, we believe that performing a TTE is a sufficient screening modality for detecting LAT in patients with AIS. While our results support prior investigations which confirmed the utility of TEE in detecting LA [18], the risk of conducting a transesophageal study is not inconsequential. Although life-threatening sequelae are rare $(<2 \%)$, intraoperative risks are notable for cardiac arrhythmias, hypoxia, hypotension, adverse response to anesthesia, and esophageal or gastric perforation while postoperative complications include odynophagia, dysphagia, and transient or permanent vocal cord dysfunction $[6,19-21]$. Our findings support that LAT is unlikely to be found on TEE if TTE shows normal LA size.

Our study is unique in that we analyzed the utility of TTE and TEE for AIS patients through a robust propensity score matching technique. This can reduce the selection bias added from choice of imaging modality, as well as the influence of possible confounding factors from an observational study design. This methodology, which was used for a similar reason by a recent multicenter study [22], can better approximate the more unbiased results seen in a randomized controlled trial. A randomized trial otherwise may not be performed due to the inherent challenges of implementing a randomized approach to diagnostic imaging for patient care. As a result, our study can be viewed as a meaningful contribution to ameliorate the gap in the literature on this topic.

Although our findings are not unexpected, the implications are considerable. By utilizing TTE to screen for LAT, providers have the opportunity to cut the complications and costs associated with routine TEE. For stroke patients in whom a left atrial thrombus is suspected, a normally sized left 
atrium on transthoracic echocardiogram may be sufficient if windows are adequate and the technician is experienced. Advances in technology and technique have improved the yield of this diagnostic modality [23], and newer devices may permit more mobile and accurate data capture [24]. Knowledge that TTE may be sufficient for predicting LAT formation may improve the confidence of providers who are considering TEE for patients at greater risk of perioperative complications.

In our study, we found that only about $71 \%$ of patients who had LAE on TTE had confirmatory LAE on TEE. Biplane axial length measurement is the recommended method of determining left atrial size on TEE; however, it often gives the largest value for the calculation for left atrial volume [25]. Although TEE is considered to be the gold standard for visualization of left atrial abnormalities [12], the appearance of thrombi formation among patients can vary $[6,26]$. Thus, intraoperator imaging and interpretation may contribute to the analyses conducted within our study. Moreover, due to the population served by the Stroke Center, a wide age range was noted in both groups involved in this study. As a result, the younger patient population in both groups may possibly preclude the efficacy of TTE to analyze cardiac dimensions or contents properly $[27,28]$, especially given the superior aspect of the cardiac chamber analyzed within this study.

While LAE and its various relationships to AIS have been reviewed throughout this report, the relationship between cardiac dimensions noted on echocardiographic imaging and clinically relevant endpoints has been recently established within the literature. For patients with AF, actual chamber size (measured in $\mathrm{cm}^{2}$ on TEE) has not been associated with the development of embolic events or AIS [29]. However, the volume to BSA ratio $\left(\mathrm{cm} / \mathrm{m}^{2}\right)$, thrombi size, and LAA size have been noted to be important factors in the formation of thrombi in AIS patients [27-29].

Our study is limited by its small sample size representing patients from a single center. As a result, the low LAT event rate may have prevented sufficient comparison between TTE and TEE parameters in predicting this outcome. There is also potential for selection bias as to which patients had both TTE and TEE performed. At our institution, the decision to perform TTE and/or TEE is individually made for each patient by the treating physician, and some patients with equivocal findings on TTE may have had TEE ordered secondarily to pursue cardioembolic causes of stroke. However, considering the limitations of a cross-sectional design, propensity score matching is a rigorous and sophisticated way of reducing selection bias as much as possible by controlling for probability of receiving TTE versus both TTE and TEE. We did not explore other TEE indications for oral anticoagulant therapy such as "smoke"/spontaneous echo contrast. For this reason, one cannot conclude that TEE has no value in the evaluation of the cause of stroke in patients without LAE on TTE. We can only conclude that LAT is unlikely to be found on TEE if TTE shows normal LA size.

In conclusion, our results may help increase provider confidence for using TTE in the AIS patient. Since TEE is highly sensitive, initial testing can be done with this modality if there are acute indications of AIS in a patient. With TTE evidence of LAE as a prerequisite for TEE, providers may detect the presence of LAT in patients with AIS in a safe and effective manner.

\section{Competing Interests}

The authors declare that they have no competing interests.

\section{References}

[1] S. Palacio and R. G. Hart, "Neurologic manifestations of cardiogenic embolism," Neurologic Clinics, vol. 20, no. 1, pp.179193, 2002.

[2] A. Arboix and J. Alió, "Cardioembolic stroke: clinical features, specific cardiac disorders and prognosis," Current Cardiology Reviews, vol. 6, no. 3, pp. 150-161, 2010.

[3] T. T. De Abreu, S. Mateus, C. Carreteiro, and J. Correia, "Therapeutic implications of transesophageal echocardiography after transthoracic echocardiography on acute stroke patients," Vascular Health and Risk Management, vol. 4, no. 1, pp. 167-172, 2008.

[4] M. D. Lesh and E. J. van der Burg, "Method and device for left atrial appendage occlusion," Google Patents, 2000.

[5] S. F. T. M. De Bruijn, W. R. P. Agema, G. J. Lammers et al., "Transesophageal echocardiography is superior to transthoracic echocardiography in management of patients of any age with transient ischemic attack or stroke," Stroke, vol. 37, no. 10, pp. 2531-2534, 2006.

[6] W. G. Daniel, R. Erbel, W. Kasper et al., "Safety of transesophageal echocardiography-a multicenter survey of 10,419 examinations," Circulation, vol. 83, no. 3, pp. 817-821, 1991.

[7] J. E. Siegler, A. K. Boehme, A. M. Dorsey et al., "A comprehensive stroke center patient registry: advantages, limitations, and lessons learned," Medical Student Research Journal, vol. 2, pp. 21-29, 2013.

[8] H. P. Adams Jr., B. H. Bendixen, L. J. Kappelle et al., "Classification of subtype of acute ischemic stroke. Definitions for use in a multicenter clinical trial. TOAST. Trial of Org 10172 in Acute Stroke Treatment," Stroke, vol. 24, no. 1, pp. 35-41, 1993.

[9] R. M. Lang, M. Bierig, R. B. Devereux et al., "Recommendations for chamber quantification: a report from the American Society of Echocardiography's guidelines and standards committee and the Chamber Quantification Writing Group, developed in conjunction with the European Association of Echocardiography, a branch of the European Society of Cardiology," Journal of the American Society of Echocardiography, vol. 18, no. 12, pp. 14401463, 2005.

[10] T. S. Tsang, W. P. Abhayaratna, M. E. Barnes et al., "Prediction of cardiovascular outcomes with left atrial size: is volume superior to area or diameter?" Journal of the American College of Cardiology, vol. 47, no. 5, pp. 1018-1023, 2006.

[11] F. E. Silvestry, M. S. Cohen, L. B. Armsby et al., "Guidelines for the echocardiographic assessment of atrial septal defect and patent foramen ovale: from the american society of echocardiography and society for cardiac angiography and interventions," Journal of the American Society of Echocardiography, vol. 28, no. 8, pp. 910-958, 2015.

[12] R. J. Lee, T. Bartzokis, T.-K. Yeoh, H. R. Grogin, D. Choi, and I. Schnittger, "Enhanced detection of intracardiac sources of cerebral emboli by transesophageal echocardiography," Stroke, vol. 22 , no. 6, pp. 734-739, 1991. 
[13] R. J. Little and D. B. Rubin, "Causal effects in clinical and epidemiological studies via potential outcomes: concepts and analytical approaches," Annual Review of Public Health, vol. 21, no. 1, pp. 121-145, 2000.

[14] I. Shrier, "Re: the design versus the analysis of observational studies for causal effects: parallels with the design of randomized trials," Statistics in Medicine, vol. 27, no. 14, pp. 2740-2742, 2008.

[15] T. Kurth, A. M. Walker, R. J. Glynn et al., "Results of multivariable logistic regression, propensity matching, propensity adjustment, and propensity-based weighting under conditions of nonuniform effect," American Journal of Epidemiology, vol. 163 , no. 3, pp. 262-270, 2006.

[16] A. Abadie and G. W. Imbens, "Large sample properties of matching estimators for average treatment effects," Econometrica. Journal of the Econometric Society, vol. 74, no. 1, pp. 235267, 2006.

[17] W. G. Cochran and D. B. Rubin, "Controlling bias in observational studies: a review," Sankhya, The Indian Journal of Statistics A, vol. 35, no. 4, pp. 417-446, 1973.

[18] W. J. Manning, R. M. Weintraub, C. A. Waksmonski et al., "Accuracy of transesophageal echocardiography for identifying left atrial thrombi: a prospective, intraoperative study," Annals of Internal Medicine, vol. 123, no. 11, pp. 817-822, 1995.

[19] T. S. M. Tsang, W. P. Abhayaratna, M. E. Barnes et al., "Prediction of cardiovascular outcomes with left atrial size: is volume superior to area or diameter?" Journal of the American College of Cardiology, vol. 47, no. 5, pp. 1018-1023, 2006.

[20] G. Côté and A. Denault, "Transesophageal echocardiographyrelated complications," Canadian Journal of Anesthesia, vol. 55, no. 9, pp. 622-647, 2008.

[21] S. Price, E. Nicol, D. G. Gibson, and T. W. Evans, "Echocardiography in the critically ill: current and potential roles," Intensive Care Medicine, vol. 32, no. 1, pp. 48-59, 2006.

[22] S.-J. Hong, J.-Y. Kim, J.-B. Kim et al., "Multidetector computed tomography may be an adequate screening test to reduce periprocedural stroke in atrial fibrillation ablation: a multicenter propensity-matched analysis," Heart Rhythm, vol. 11, no. 5, pp. 763-770, 2014.

[23] J. Hung, R. Lang, F. Flachskampf et al., "3D echocardiography: a review of the current status and future directions," Journal of the American Society of Echocardiography, vol. 20, no. 3, pp. 213233, 2007.

[24] M. J. Liebo, R. L. Israel, E. O. Lillie, M. R. Smith, D. S. Rubenson, and E. J. Topol, "Is pocket mobile echocardiography the next-generation stethoscope? A cross-sectional comparison of rapidly acquired images with standard transthoracic echocardiography," Annals of Internal Medicine, vol. 155, no. 1, pp. 33-38, 2011.

[25] P. Jiamsripong, T. Honda, C. S. Reuss et al., "Three methods for evaluation of left atrial volume," European Heart JournalCardiovascular Imaging, vol. 9, no. 3, pp. 351-355, 2008.

[26] I. W. Black, C. N. Chesterman, A. P. Hopkins, L. C. L. Lee, B. H. Chong, and W. F. Walsh, "Hematologic correlates of left atrial spontaneous echo contrast and thromboembolism in nonvalvular atrial fibrillation," Journal of the American College of Cardiology, vol. 21, no. 2, pp. 451-457, 1993.

[27] Y. Agmon, B. K. Khandheria, I. Meissner et al., "Left atrial appendage flow velocities in subjects with normal left ventricular function," American Journal of Cardiology, vol. 86, no. 7, pp. 769-773, 2000.
[28] M. Pepi, A. Evangelista, P. Nihoyannopoulos et al., "Recommendations for echocardiography use in the diagnosis and management of cardiac sources of embolism," European Journal of Echocardiography, vol. 11, no. 6, pp. 461-476, 2010.

[29] T. S. M. Tsang, M. E. Barnes, B. J. Gersh, K. R. Bailey, and J. B. Seward, "Left atrial volume as a morphophysiologic expression of left ventricular diastolic dysfunction and relation to cardiovascular risk burden," American Journal of Cardiology, vol. 90, no. 12, pp. 1284-1289, 2002. 


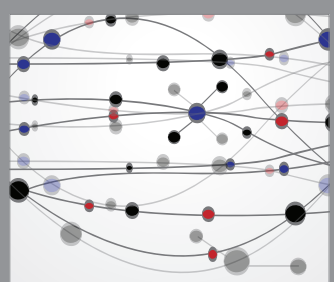

The Scientific World Journal
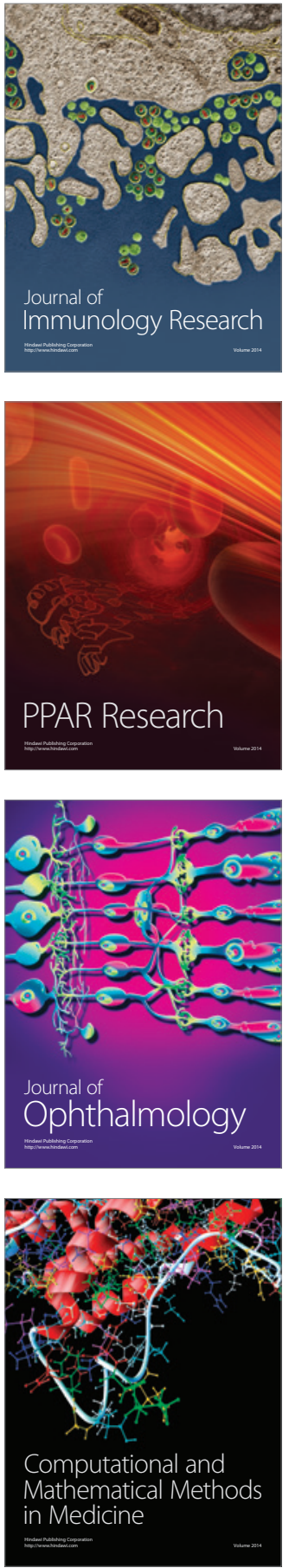

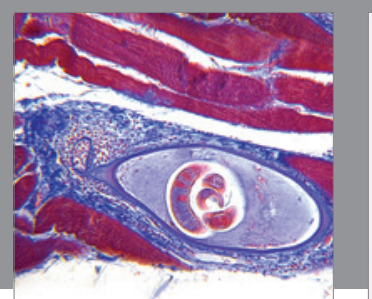

Gastroenterology Research and Practice

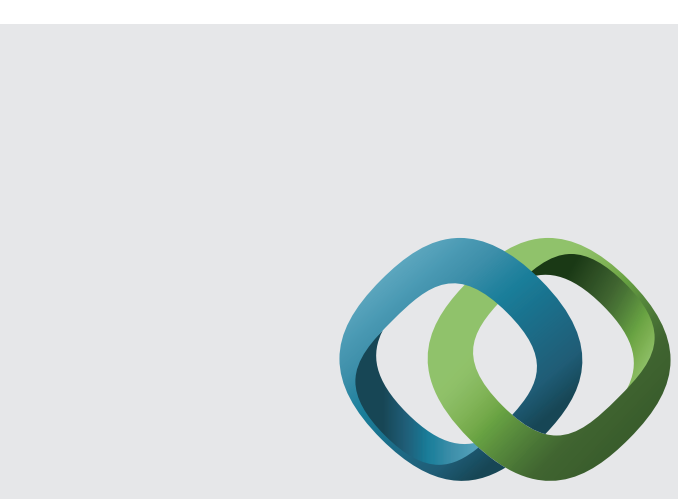

\section{Hindawi}

Submit your manuscripts at

http://www.hindawi.com
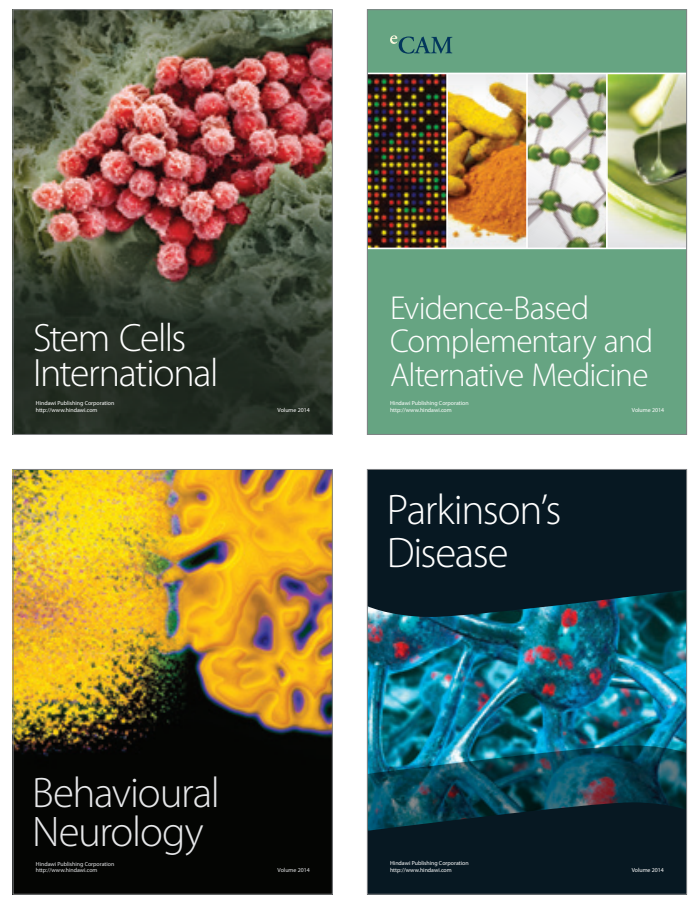
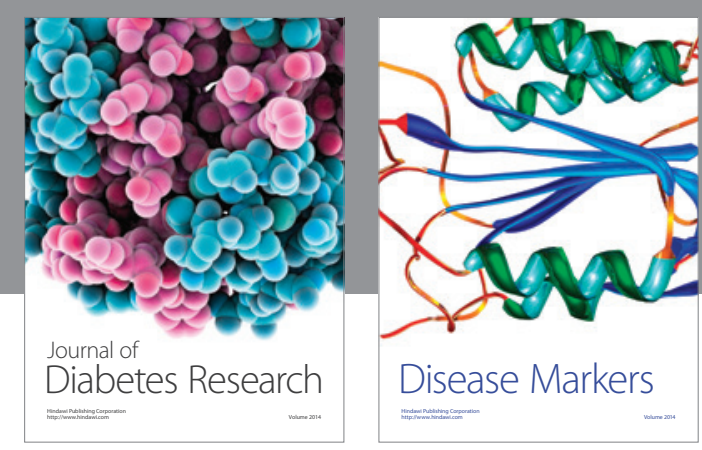

Disease Markers
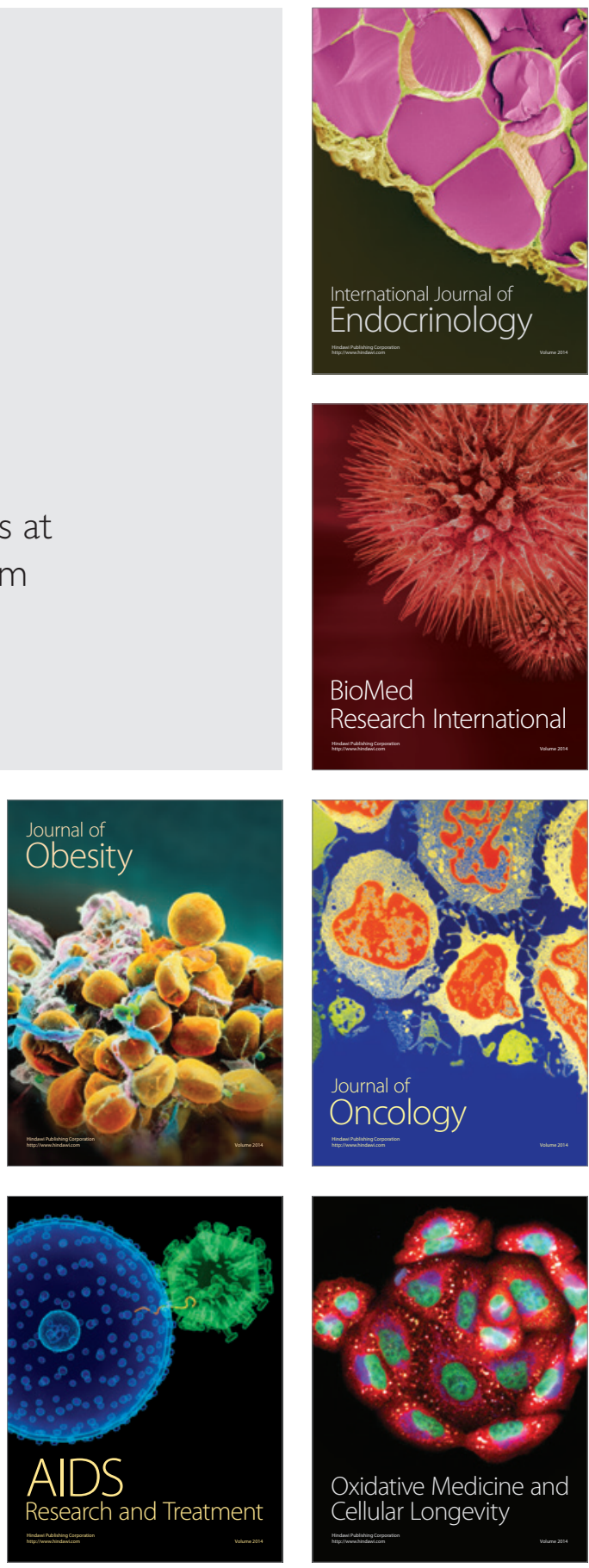